\title{
Comparison of different methods of heart rate entropy analysis during acute anoxia superimposed on a chronic rat model of pulmonary hypertension
}

\author{
Hernâni Gonçalves ${ }^{a, *}$, Tiago Henriques-Coelho ${ }^{b}$, Ana Paula Rocha ${ }^{c, d}$, André P. Lourenço ${ }^{b}$, \\ Adelino Leite-Moreira ${ }^{\mathrm{b}}$, João Bernardes ${ }^{\mathrm{a}, \mathrm{e}, \mathrm{f}}$ \\ a Center for Research in Health Technologies and Information Systems (CINTESIS), Faculty of Medicine, University of Porto, Al. Prof. Hernâni Monteiro, 4200-319 Porto, Portugal \\ ${ }^{b}$ Department of Physiology and Cardiothoracic Surgery, Faculty of Medicine, University of Porto, Al. Prof. Hernâni Monteiro, 4200-319 Porto, Portugal \\ c Departamento de Matemática, Faculdade de Ciências, Universidade do Porto, Rua do Campo Alegre, s/n, 4169-007 Porto, Portugal \\ d Centro de Matemática da Universidade do Porto, Rua do Campo Alegre, s/n, 4169-007 Porto, Portugal \\ e Department of Obstetrics and Gynecology, Faculty of Medicine, University of Porto, Al. Prof. Hernâni Monteiro, $4200-319$ Porto, Portugal \\ f INEB - Instituto de Engenharia Biomédica, Rua Dr. Roberto Frias, 4200-465 Porto, Portugal
}

\section{A R T I C L E I N F O}

\section{Article history:}

Received 9 December 2011

Received in revised form 19 June 2012

Accepted 27 June 2012

\section{Keywords:}

Heart rate variability (HRV)

Time and frequency domain analysis

Entropy

Anoxia

\begin{abstract}
A B S T R A C T
Acute life-threatening situations are particularly critical when superimposed on chronic diseases. The objective of this study was the assessment of heart rate (HR) dynamics during episodes of acute anoxia superimposed on a rat model of chronic pulmonary hypertension. In 10 adult Wistar rats, five weeks after pulmonary hypertension induction with Monocrotaline, we analysed eight 1-min HR segments, during episodes of baseline, mechanical ventilation and acute anoxia, using linear indices, approximate entropy (ApEn), sample entropy (SampEn) and multiscale entropy (MSE). The transition from baseline or mechanical ventilation to early anoxia was identified through almost all indices, but $\operatorname{SampEn}(2,0.6)$ was the index that better identified all the transitions. MSE presented limited performance, possibly due to the non-stationary nature and short duration of the acute anoxia episodes. A systematic evaluation of all computed HR indices may help to identify which indices or combination of indices more adequately discriminates and monitors critical acute events superimposed on chronic clinical conditions.
\end{abstract}

(c) 2012 IPEM. Published by Elsevier Ltd. All rights reserved.

\section{Introduction}

Clinicians are often confronted with acute life-threatening situations superimposed on chronic diseases, both during pre and postnatal life, such as acute anoxia during labour superimposed on a severely growth retarded fetus or acute myocardial infarction superimposed on a chronic heart failure condition. Accordingly, intensive monitoring of patients with severe chronic conditions is mandatory when they suffer, or are at high risk of suffering, an acute insult, namely during labour, surgery or intensive care treatment. In this setting, heart rate (HR) analysis remains as one of the most used and useful monitoring tools.

Linear methods for HR variability (HRV) analysis, in both time and frequency domains, have been applied in human studies and standard guidelines were established [41]. Spectral analysis, in particular, allows the identification of two distinct peaks, linked to the activities of the two branches of the autonomic nervous system [41]. However, the application of these methods to animal studies,

\footnotetext{
* Corresponding author. Tel.: +351 225513622; fax: +351 225513601.

E-mail addresses: hernanigoncalves@med.up.pt, hernani.goncalves@fc.up.pt (H. Gonçalves).
}

needs to be adapted to every situation [2], considering results heralded from previous research, that may be used as guidelines, although it should be noted that different experimental settings may lead to significant differences in HRV parameters.

Nonlinear methods have also been applied to HRV analysis [1,2]. The two main categories of these methods are: (i) indices that describe the scaling behaviour of a system; (ii) indices that describe the complexity of a system. The scaling behaviour of a system may be evaluated through fractal dimension (FD), $1 / f$ slope or detrended fluctuation analysis (DFA). The analysis of a system in the phase space enables the assessment of its complexity. The correlation dimension indicates the dimension of the phase space, Lyapunov exponents provide the sensitivity of the system to initial conditions and approximate entropy (ApEn) is a measure of the system complexity [2].

ApEn [34,36] has been widely applied in both human $[17,18,23,40]$ and animal studies [19,20,32]. Nevertheless, the selection of ApEn parameters may significantly influence the analysis between normal and heart failure human groups [29]. Indeed, although reference values for the embedding dimension $m$ and the threshold $r$ have been proposed [36], recent work underline the critical role of parameter choice, particularly the threshold $r[7,30]$. Therefore, research on this topic is required, namely pertaining to 
the application of ApEn to animal studies. Moreover, traditional entropy-based measures yield higher values for systems exhibiting long-range correlations than for random systems, such as in the case of white noise and for $1 / f$ noise. These contradictory results led Costa et al. to propose the multiscale entropy (MSE) analysis, a new entropy-based method which incorporates both entropy and scale $[6,8,11]$. This method may be more appropriate for a correct estimation of system complexity and also enables the distinction between synthetic and physiological time series [9], since synthetic time series are simpler than physiologic time series, which under healthy conditions, present a complex temporal structure with multiscale correlations. However, little is known about the applicability of MSE analysis to animal studies [5,43].

The objective of this study was the assessment of HR dynamics with linear and nonlinear methods during episodes of acute anoxia superimposed in a rat model of chronic pulmonary hypertension, with particular emphasis on common and novel entropy-based methods.

\section{Methods}

\subsection{Animal model}

Pulmonary hypertension was induced, with a single subcutaneous $60 \mathrm{mg} / \mathrm{kg}$ Monocrotaline injection (Sigma, Barcelona, Spain), in 10 adult female Wistar rats (Charles River Laboratories; Barcelona, Spain), weighing 215-260 g, with ages ranging between 6 and 8 weeks, previously used in another study [20], were housed in groups of 5 rats/cage, in a controlled environment, under a 12:12$\mathrm{h}$ light-dark cycle, at a room temperature of $22^{\circ} \mathrm{C}$, with a free supply of food and water. All rats initially had ECG monitoring during $20 \mathrm{~min}$ (week 0 ) under ketamine anaesthesia $(50 \mathrm{mg} / \mathrm{kg}$, intraperitonealy) and were subsequently monitored in a similar way weekly for 5 weeks (weeks 1-5). By the end of the third week, one rat died and the other rats presented signs of overt heart failure, namely lethargy, laboured breathing, cachexia, vein and liver engorgement, pleural effusion and ascites, as well as significant changes in linear and nonlinear HR indices [20]. In the last week (week 5), after $20 \mathrm{~min}$ of acquisition, they were also mechanically ventilated with $100 \% \mathrm{O}_{2}$ at a respiratory rate of $1.25 \mathrm{~Hz}$ and a tidal volume of $2 \mathrm{~mL}$, during $1 \mathrm{~min}$ and then with $5 \% \mathrm{CO}_{2}$ and $95 \% \mathrm{~N}_{2}$ during $5 \mathrm{~min}$ of induced anoxia. All animal experiments were performed according to the Portuguese law on animal welfare and the National Institutes of Health Guide for the Care and Use of Laboratory Animals (NIH Pub. No. 85-23, Revised 1996).

\subsection{Heart-rate acquisition and pre-processing}

HR acquisition and pre-processing was performed as described elsewhere $[19,20]$. Shortly, one electrode was placed subcutaneously in each leg, to acquire the ECG signal at a sampling rate of $500 \mathrm{~Hz}$, according to a standardized procedure [19]. The tachogram obtained after automatic $\mathrm{R}$ wave detection and expert validation was subsequently resampled at a frequency of $8 \mathrm{~Hz}$ - in order to provide a correct coverage of the considered spectral bands in HR analysis - and then converted to the HR signal, in beats per minute (bpm), using cubic spline interpolation. Due to experimental difficulties already mentioned in Section 2.1 and to some periods of signal loss, only some segments were available for analysis: 8 in the baseline period, 6 during mechanical ventilation and 7, 6 and 7 segments in the first, second and third minutes of induced anoxia, respectively. In order to provide some insight on the occurrence rate of ectopic beats, HR values which are $10 \%$ above or below from their previous HR value were considered as potentially being associated with an ectopic beat. Accordingly, the average of this occurrence rate was found to be $0.0 \%$ in the baseline, $0.4 \%$ during mechanical ventilation and around $2.2 \%$ in the anoxia period.

\subsection{Heart rate analysis}

Eight 1-min segments (each segment corresponding to 480 points) without signal loss (signal loss was manually identified) were analysed: the first 4 min during baseline recording (excluding the first $2 \mathrm{~min}$ in order to ensure a stable tracing) assigned
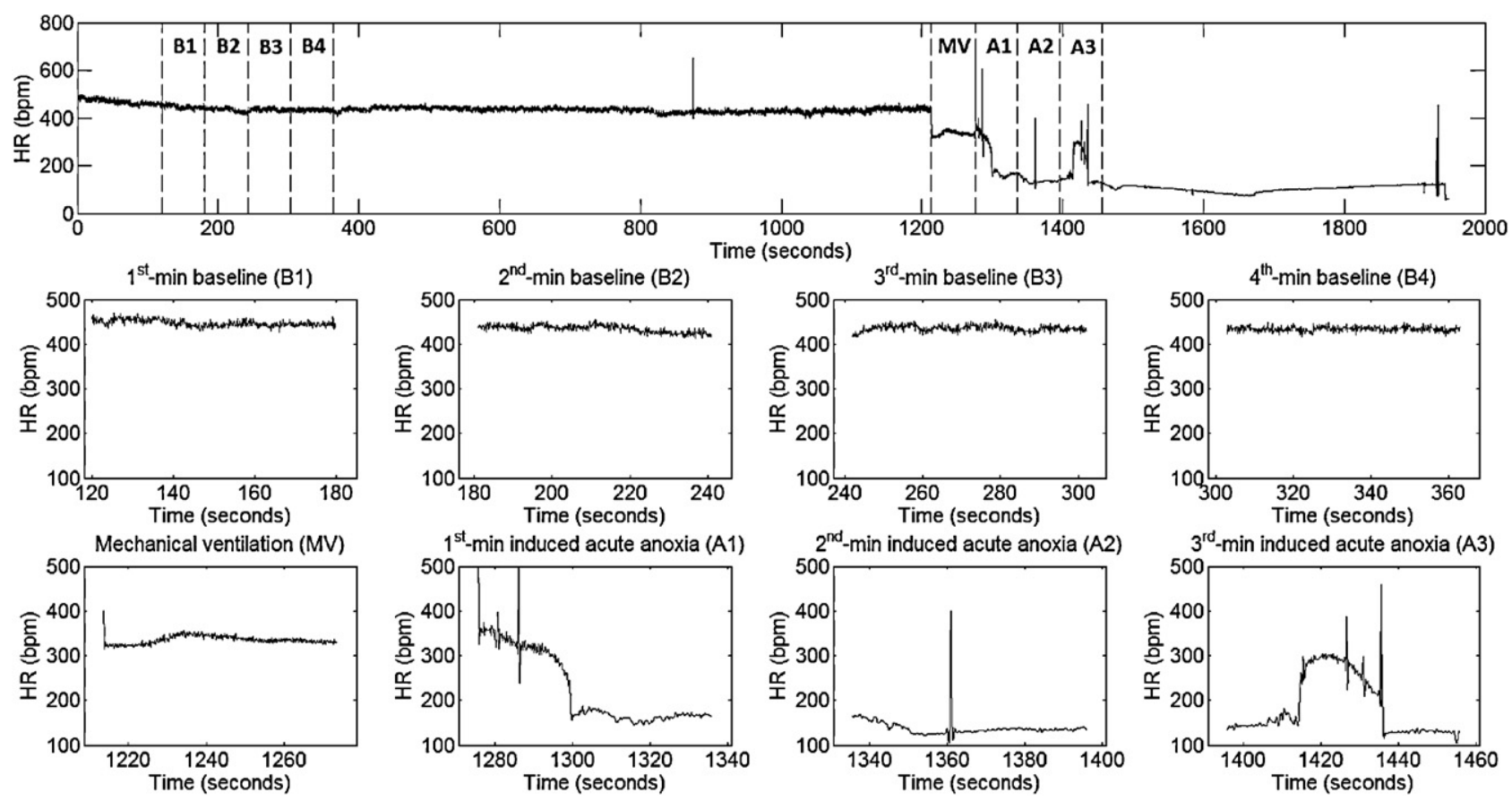

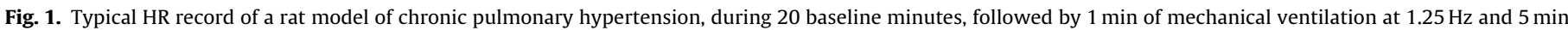

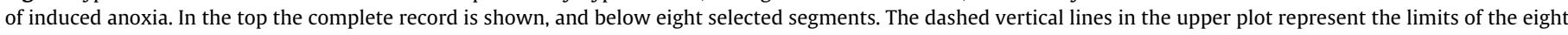
selected segments represented in the lower plots. 
respectively B1, B2, B3 and B4; 1 min during mechanical ventilation assigned MV; and the first 3 min of induced anoxia assigned respectively A1, A2 and A3. An example of a full HR signal and its respective eight segments is presented in Fig. 1. The spikes visible in Fig. 1 correspond to ectopic beats, since errors motivated by the automatic detection of the $\mathrm{R}$ wave were manually corrected as mentioned in Section 2.2. The ectopic beats were not eliminated, particularly because they may represent a 'typical' pattern in anoxia periods - rather than isolated points present in normal recordings of HRV (animal or adult) - avoiding the loss of important information.

HR variability analysis was based on both linear (time- and frequency domain) and nonlinear (entropy) indices. Particular attention is devoted to nonlinear entropy-based analysis. The considered indices are described in the following sections.

\subsubsection{Time- and frequency domain (linear) indices}

Time- and frequency-domain measure analysis was carried out as previously described [16]. Time-domain measures included mean HR (mHR) and respective standard deviation (sdHR), longterm irregularity (LTI), mean amplitude between maximum and minimum in each minute $(\Delta)$, short-term variability (STV) and interval index (II). All these indices with the exception of II index reflect gross changes in FHR average and variability, whereas the II index assesses short-term FHR variability taking into account long-term variability. Further details may be found in [16].

For spectral data characterization, non-parametric (Welch) spectrum estimation was performed [22], considering a Hanning window of 256 points and $62.5 \%$ of superposition. Several spectral bands have been described and analysed for rat HR [3,26]. The decomposition of HRV allows the identification of a very low frequency component (VLF), below $0.04 \mathrm{~Hz}$, and two components associated to the low and high frequencies (LF and HF), centred approximately at $0.1 \mathrm{~Hz}(0.04-1.00 \mathrm{~Hz})$ and at the respiratory frequency $(1.00-3.00 \mathrm{~Hz})$, respectively [26]. These spectral bands were previously validated [20].

\subsubsection{Entropy analysis}

Entropy is a concept that addresses system randomness or predictability, with greater entropy reflecting higher randomness and lower system order [35]. In addition, entropy has been shown to be a parameter that characterizes chaotic behaviour [39]. There are several distinct entropy formulations. The classical Shannon's entropy [38], $H(X)$, of a given signal $X$, is defined as

$$
H(X)=-\sum_{x_{i}} p\left(x_{i}\right) \log p\left(x_{i}\right)=-E\left[\log p\left(x_{i}\right)\right]
$$

where $X$ represents a single discrete random variable a set of values $\Theta$ and probability mass function $p\left(x_{i}\right)=P_{r}\left\{X=x_{i}\right\}, x_{i} \in \Theta$ and $E$ represents the expectation operator. The Kolmogorov-Sinai (K-S) entropy, developed by Kolmogorov [25] and later expanded by Sinai, was the basis of the development of some entropy algorithms, such as those given by Grassberger and Procaccia [21] and by Eckmann and Ruelle [13]. However, the KS entropy was not developed for statistical applications, presenting several limitations in this regard.

\subsubsection{Approximate entropy (ApEn) and sample entropy (SampEn).} In order to provide a wide applicability of an entropy measure, Pincus [34] proposed approximate entropy (ApEn), which was constructed along thematically similar lines to the K-S entropy. It allows for addressing data length constraints commonly encountered in several applications such as in the analysis of the HR signal, and is potentially applicable to noisy time-series data [36]. Given $N$ data points $\{x(i)\}$ from a HR signal, a sequence of $N-m+1$ vectors $\{u(i)\}$ is formed by $u(i)=[x(i), \ldots, x(i+m-1)]$, where $m$ corresponds to each vector length. Based on the distance $d[u(i), u(j)]$, defined as the maximum difference between vectors $u(i)$ and $u(j)$ respective scalar components (i.e., the distance is the $L_{\infty}$-norm), the quantity

$C_{i}^{m}(r)=\frac{\text { number of } j \leq N-m+1 \text { such that } d[u(i), u(j)] \leq r}{N-m+1}$

measures the frequency of patterns similar to a given pattern of window length $m$ within a tolerance $r$. The average of the natural logarithm of the $C_{i}^{m}(r)$ values is defined as

$\phi^{m}(r)=\frac{1}{N-m+1} \sum_{i=1}^{N-m+1} \ln \left(C_{i}^{m}(r)\right)$

Then $\operatorname{ApEn}(m, r)$ is defined as

$\operatorname{ApEn}(m, r)=\lim _{N \rightarrow \infty}\left[\phi^{m}(r)-\phi^{m+1}(r)\right]$

and based on a finite number of $N$ data points it can be estimated by the family of statistics

$\operatorname{ApEn}(m, r, N)=\phi^{m}(r)-\phi^{m+1}(r)$

Pincus and Goldberger [35] have stated that ApEn decrease often correlates with standard deviation (SD) decrease. However, they suggest that it may be possible to decorrelate ApEn from SD, by specifying $r$ in $\operatorname{ApEn}(m, r, N)$ as a fixed percentage of the sample SD of the individual subject data set. They call this normalized regularity. Based on calculations that included both theoretical analysis and clinical applications, $m=1$ or 2 and $r$ between 0.1 and $0.25 \times \mathrm{SD}$ of the $x(i)$ data produce good statistical validity of $\operatorname{ApEn}(m, r, N)$ [36]. Based on previous works $[19,20], m$ was considered as 2 and the set of values 0.1 SD, 0.15 SD, 0.2 SD, 0.6 SD, 0.9 SD and 1.2 SD was considered for parameter $r$. A fast algorithm for ApEn computation is proposed in [14]. Despite the $\operatorname{ApEn}(m, r)$ algorithm similarities, $\operatorname{ApEn}(m, r, N)$ is not intended to be an approximate value of the $\mathrm{K}-\mathrm{S}$ entropy [34,35]. ApEn has several technical advantages in comparison to K-S entropy for statistical usage: it is nearly unaffected by noise of magnitude below $r$, the filter level; it is robust to occasional artifacts, either large or small; it gives meaningful information with a reasonable number of data points; and it is finite for both stochastic and deterministic processes. Therefore, ApEn is applicable to noisy, medium-sized data sets, such as those typically encountered in HR data analysis [35].

$\operatorname{ApEn}(m, r, N)$ is a biased statistic of $\operatorname{ApEn}(m, r)$, as the expected value of $\operatorname{ApEn}(m, r, N)$ is less than the parameter $\operatorname{ApEn}(m, r)$. This is mainly due to the fact that ApEn counts self-matching's. Sample entropy (SampEn), proposed by Richman and Moorman [37], is an entropy measure which is computed in a similar manner as ApEn, with the major difference that SampEn does not count selfmatches, having reduced bias when compared to ApEn. Despite the advantages of SampEn over ApEn, in some cases ApEn has shown to provide better discrimination ability than SampEn, and thus both measures were considered in the present work. The same set of values for parameters $m$ and $r$ was considered for ApEn and SampEn.

For the sake of simplicity, hereafter $\operatorname{ApEn}(m, r, N)$ and Sam$\operatorname{pEn}(m, r, N)$ will be merely assigned as $\operatorname{ApEn}(m, r)$ and $\operatorname{SampEn}(m, r)$, as all the analysed segments are composed by 480 points (as previously described).

2.3.2.2. Errors in the estimation of ApEn and SampEn due to the finite resolution of the $R R$ time series. The quantification errors in the discrete RR time series produce considerable errors in the ApEn estimation (bias and variance) when the signal variability or the sampling frequency is low [15]. García-González et al. [15] proposed a figure of merit of the RR time series [signal to resolution 

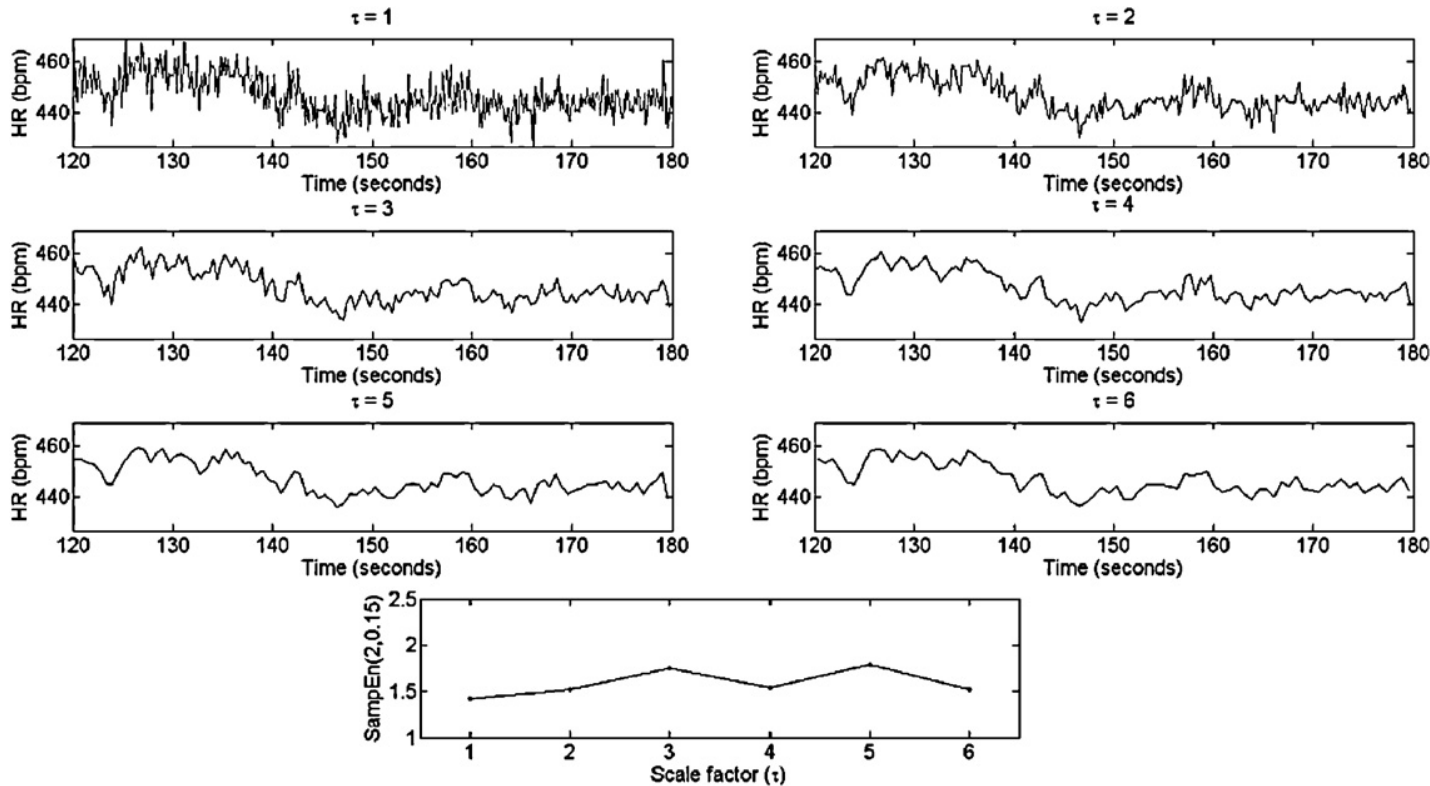

Fig. 2. Example of the 1-min baseline segment showed in Fig. 1 in scales 1-6 and corresponding SampEn(2,0.15) values of each scale according to the MSE analysis.

of the neighbourhood ratio (SRN)] in the computation of ApEn (or any other quantification of the recurrence plot) defined as

$S R N=S D N N \times f_{S} \times r=\varepsilon f_{S}$

where $S D N N$ is the standard deviation of the RR sequence, $f_{S}$ is the sampling frequency of the ECG and $r$ corresponds to the threshold parameter previously described. This figure of merit allows for relating SRN with the errors associated with ApEn computation in two ways:

- It is expected that a time series with SRN near an integer number will have greater errors than those with SRN near $n+1 / 2(n \in \aleph)$;

- The greater the SRN, the lower the error (not monotonically).

Further details supporting these observations are found elsewhere [15]. Based on their results, it could be roughly inferred that in order to achieve an error less than 0.1 in ApEn computation, the SRN measure should be approximately greater than 3 regardless of its fluctuations around integer numbers [15]. In this work the HR signal is analysed instead of the RR sequence, followed by a resampling procedure. We evaluated the figure of merit SRN prior to the conversion of the RR sequence to HR signal. This allows for evaluating whether the sampling frequency of the ECG leads to "acceptable" errors in ApEn computation.

2.3.2.3. Criteria for the selection of the threshold parameter $r$. The selection of the threshold parameter $r$ in ApEn and SampEn is controversial and still a subject of research $[7,29,30]$. Lu et al. showed that one should use the parameter $r$ corresponding to the maximum ApEn value, in order to discriminate time series with different levels of complexity [30]. However, the search for such parameter is a computationally costly task. Therefore, they proposed general formulas for the selection of parameter $r$ for each individual, instead of a fixed percentage. The equations for embedding dimension $m=2$ proposed by Lu et al. [30] and Chon et al. [7], which relies on the selection of the threshold $r$, are respectively

$r_{\mathrm{Lu}}=\frac{-0.02+0.23 \sqrt{s d_{1} / s d_{2}}}{\sqrt[4]{N / 1000}}$ $r_{\text {Chon }}=\frac{-0.036+0.26 \sqrt{s d_{1} / s d_{2}}}{\sqrt[4]{N / 1000}}$

The quantity $s d_{1}$ corresponds to the short-time variability and is computed as the standard deviation of the successive differences of $\{x(i)\}$, whereas $s d_{2}$ is associated to long-term variability of the signal and is computed as the standard deviation of $\{x(i)\}$. These two formulas for the selection of parameter $r$ were considered in addition to the previously referred set of values for $r$.

2.3.2.4. Multiscale entropy (MSE) analysis. Costa et al. proposed a new entropy-based method, multiscale entropy (MSE) analysis, which incorporates entropy and scale [8,11]. Given a onedimensional discrete time series, $\left\{x_{1}, \ldots, x_{i}, \ldots x_{N}\right\}$, coarse-grained time-series are constructed as

$y_{j}^{(\tau)}=\frac{1}{\tau} \sum_{i=(j-1) \tau+1}^{j \tau} x_{i}, \quad 1 \leq j \leq \frac{N}{\tau}$

where $\tau$ stands for the scale factor. It consists on dividing the original time series into nonoverlapping windows of length $\tau$, following by the averaging of the data points inside each window. Finally, an entropy measure (usually SampEn) is calculated for each coarsegrained time series plotted as a function of the scale factor $\tau$. This procedure is called MSE analysis. Since in this work 1-min segments are considered, which are composed by 480 points, and ApEn should be computed in segments with at least 75 points [36], the MSE analysis is performed on scales 1-6. An example of a 1-min baseline segment in scales 1-6 is provided in Fig. 2, together with the MSE analysis for $\operatorname{SampEn}(2,0.15)$.

\subsection{Statistical analysis}

The first four 1-min segments from the baseline period, the 1min segment of mechanical ventilation and the first three 1-min segments from the anoxia period (totalizing eight 1-min segments), were compared regarding each of the considered HRV indices. The measurement of the same individual at different time instants requires the use of paired approaches for hypothesis testing. However, due to signal loss in some segments of different rats and other factors previously mentioned in Sections 2.1 and 2.2, there 
Table 1

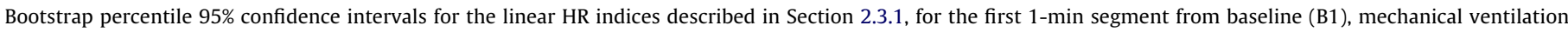

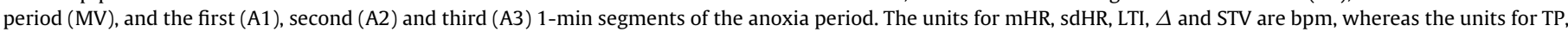
LF and HF are bpm². (a) $p<0.05$ B1 versus MV; (b) $p<0.05$ B1 versus A1; (c) $p<0.05$ MV versus A1; (d) $p<0.05$ A1 versus A2.

\begin{tabular}{|c|c|c|c|c|c|c|}
\hline & B1 & MV & A1 & $\mathrm{A} 2$ & A3 & $p$ \\
\hline mHR & $416.8-464.5$ & $243.8-411.8$ & $196.5-274.7$ & 131.1-157.9 & 109.9-180.7 & a,b,d \\
\hline sdHR & $4.9-8.0$ & $5.9-18.7$ & $41.4-81.1$ & $7.2-71.4$ & $7.4-56.2$ & $\mathrm{~b}, \mathrm{c}$ \\
\hline LTI & $7.8-12.5$ & $4.2-13.7$ & $74.0-214.6$ & $11.6-55.9$ & $15.9-149.5$ & $\mathrm{~b}, \mathrm{c}, \mathrm{d}$ \\
\hline$\Delta$ & $29.0-44.4$ & $52.7-405.9$ & $242.2-514.0$ & $25.4-1654.0$ & $24.9-355.3$ & $a, b$ \\
\hline STV & $4.5-5.4$ & $1.9-7.4$ & $6.6-14.1$ & $1.9-11.2$ & $1.3-29.1$ & $\mathrm{~b}, \mathrm{c}$ \\
\hline II & $0.71-1.01$ & $0.47-0.96$ & $0.17-0.31$ & $0.23-0.56$ & $0.15-0.53$ & $\mathrm{~b}, \mathrm{c}, \mathrm{d}$ \\
\hline TP & $23.4-40.7$ & $5.2-65.5$ & $177.0-1054.0$ & $12.6-10747.0$ & $3.9-2480.0$ & $\mathrm{~b}, \mathrm{c}$ \\
\hline LF & $2.6-7.2$ & $1.6-7.9$ & $58.5-564.3$ & $9.7-1988.0$ & $1.5-1907.0$ & $\mathrm{~b}, \mathrm{c}$ \\
\hline $\mathrm{HF}$ & $9.4-16.3$ & $2.6-44.0$ & $13.7-146.0$ & $0.1-3500.0$ & $0.0-355.3$ & \\
\hline $\mathrm{LF} / \mathrm{HF}$ & $0.2-0.8$ & $0.1-0.9$ & $0.7-34.3$ & $1.4-93.3$ & $5.4-179.4$ & $\mathrm{~b}$ \\
\hline
\end{tabular}

Table 2

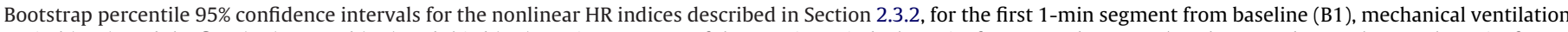

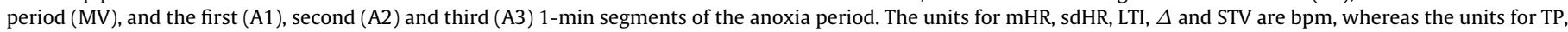
LF and HF are bpm². (a) $p<0.05$ B1 versus MV; (b) $p<0.05$ B1 versus A1; (c) $p<0.05$ MV versus A1; (d) $p<0.05$ A1 versus A2.

\begin{tabular}{|c|c|c|c|c|c|c|}
\hline & B1 & MV & A1 & $\mathrm{A} 2$ & A3 & $p$ \\
\hline $\operatorname{ApEn}(2,0.1)$ & $0.75-0.81$ & $0.79-1.06$ & $0.17-0.44$ & $0.29-0.51$ & $0.19-0.36$ & $\mathrm{~b}, \mathrm{c}$ \\
\hline $\operatorname{ApEn}(2,0.15)$ & $0.98-1.07$ & $0.76-1.06$ & $0.10-0.35$ & $0.22-0.42$ & $0.12-0.29$ & $b, c$ \\
\hline $\operatorname{ApEn}(2,0.2)$ & $1.05-1.21$ & $0.59-1.18$ & $0.09-0.25$ & $0.18-0.35$ & $0.08-0.23$ & $\mathrm{~b}, \mathrm{c}$ \\
\hline $\operatorname{SampEn}(2,0.1)$ & $1.37-1.69$ & $0.74-1.81$ & $0.14-0.36$ & $0.13-0.40$ & $0.14-0.28$ & $\mathrm{~b}, \mathrm{c}$ \\
\hline SampEn(2,0.15) & $1.31-1.61$ & $0.67-1.64$ & $0.09-0.25$ & $0.11-0.31$ & $0.09-0.17$ & $b, c$ \\
\hline $\operatorname{SampEn}(2,0.2)$ & $1.27-1.47$ & $0.55-1.49$ & $0.07-0.17$ & $0.09-0.27$ & $0.07-0.13$ & $\mathrm{~b}, \mathrm{c}$ \\
\hline $\operatorname{ApEn}(2,0.6)$ & $1.02-1.13$ & $0.04-0.85$ & $0.03-0.06$ & $0.05-0.10$ & $0.02-0.08$ & a,b \\
\hline $\operatorname{ApEn}(2,0.9)$ & $0.61-0.84$ & $0.00-0.58$ & $0.02-0.05$ & $0.03-0.07$ & $0.01-0.06$ & $\mathrm{a}, \mathrm{b}$ \\
\hline $\operatorname{ApEn}(2,1.2)$ & $0.38-0.54$ & $0.00-0.41$ & $0.01-0.05$ & $0.02-0.05$ & $0.01-0.05$ & $\mathrm{~b}$ \\
\hline SampEn(2,0.6) & $0.93-1.13$ & $0.03-0.82$ & $0.02-0.04$ & $0.04-0.09$ & $0.01-0.05$ & $\mathrm{a}, \mathrm{b}, \mathrm{c}, \mathrm{d}$ \\
\hline $\operatorname{SampEn}(2,0.9)$ & $0.52-0.77$ & $0.00-0.53$ & $0.01-0.03$ & $0.02-0.05$ & $0.01-0.04$ & $\mathrm{a}, \mathrm{b}, \mathrm{d}$ \\
\hline $\operatorname{SampEn}(2,1.2)$ & $0.33-0.49$ & $0.00-0.37$ & $0.01-0.03$ & $0.02-0.03$ & $0.01-0.03$ & $\mathrm{~b}$ \\
\hline$r_{\mathrm{Lu}}$ & $0.16-0.20$ & $0.13-0.20$ & $0.05-0.09$ & $0.04-0.18$ & $0.02-0.10$ & $\mathrm{~b}, \mathrm{c}$ \\
\hline$A p E n_{L u}$ & $1.03-1.16$ & $0.71-1.16$ & $0.33-0.49$ & $0.22-0.55$ & $0.34-0.47$ & $\mathrm{~b}, \mathrm{c}$ \\
\hline SampEn $_{\mathrm{Lu}}$ & $1.26-1.51$ & $0.65-1.56$ & $0.17-0.48$ & $0.13-0.67$ & $0.34-0.47$ & b,c \\
\hline
\end{tabular}

are non-systematic absences of recordings for certain segments, which does not allow for performing a consistent paired analysis. Therefore, the confidence intervals and hypothesis were performed without considering pairing. Given the small size of the dataset, bootstrap percentile 95\% confidence intervals for the median [31] were computed for the eight considered segments. The differences between these segments were evaluated through the nonparametric Mann-Whitney and Kruskal-Wallis statistical tests [12].

\section{Results}

In order to evaluate whether the baseline may be considered a stable segment, the first four 1-min segments from the baseline period were compared regarding each of the considered HRV indices. For all of them, no significant differences were found between the four 1 -min baseline segments $(\alpha=0.05)$. Therefore, only the results regarding the first 1-min segment from the baseline period are provided in Tables 1 and 2 . No significant differences were found between the second and third minutes of the anoxia period regarding both linear and nonlinear indices $(\alpha=0.05)$, and thus only the results for the first two 1-min segments from the anoxia period are compared through hypothesis testing in Tables 1 and 2.

The mean \pm SD for SRN regarding the eight considered HR segments for the threshold $r$ value of 0.2 , is provided in Fig. 3. It can be observed that considerable low SRN values were obtained, particularly for the HR baseline segments. The higher SRN values for the anoxia segments may be explained by two reasons: a decrease in the mean heart rate observed in these segments thus higher values of RR intervals - for which a sampling period of $0.002(500 \mathrm{~Hz})$ of the ECG becomes a relative better resolution in these segments; and a higher standard deviation, motivated by changes in the baseline. For the other considered higher $r$ values of $0.6,0.9$ and 1.2 , the figure of merit SRN will clearly be higher as expected according to Eq. (6). Despite the extremely low SRN values for the baseline segments, the RR sequence which was used for the computation of the HR indices passed through a cubic spline interpolation procedure, at a sampling frequency of $8 \mathrm{~Hz}$, which artificially minimized the quantization effects (Fig. 4). Moreover, the analysis of the SRN values was not performed considering their absolute values, as human and animal HR recordings present completely different mean and standard deviation measures.

As previously reported [19], the $0.6,0.9$ and $1.2 r$ parameter values, along with mHR and $\Delta$ were the only HR parameters that allowed the distinction between baseline and mechanical

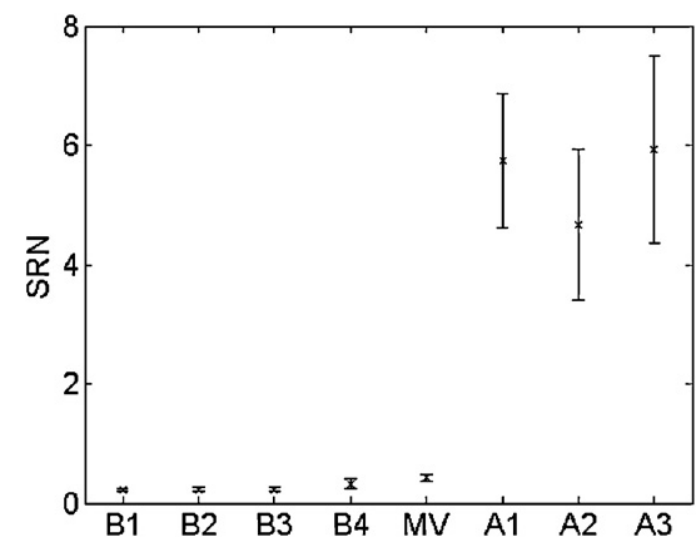

Fig. 3. Mean $\pm S D$ for the SRN figure of merit regarding the eight considered $H R$ segments, considering the threshold $r$ as 0.2 . 

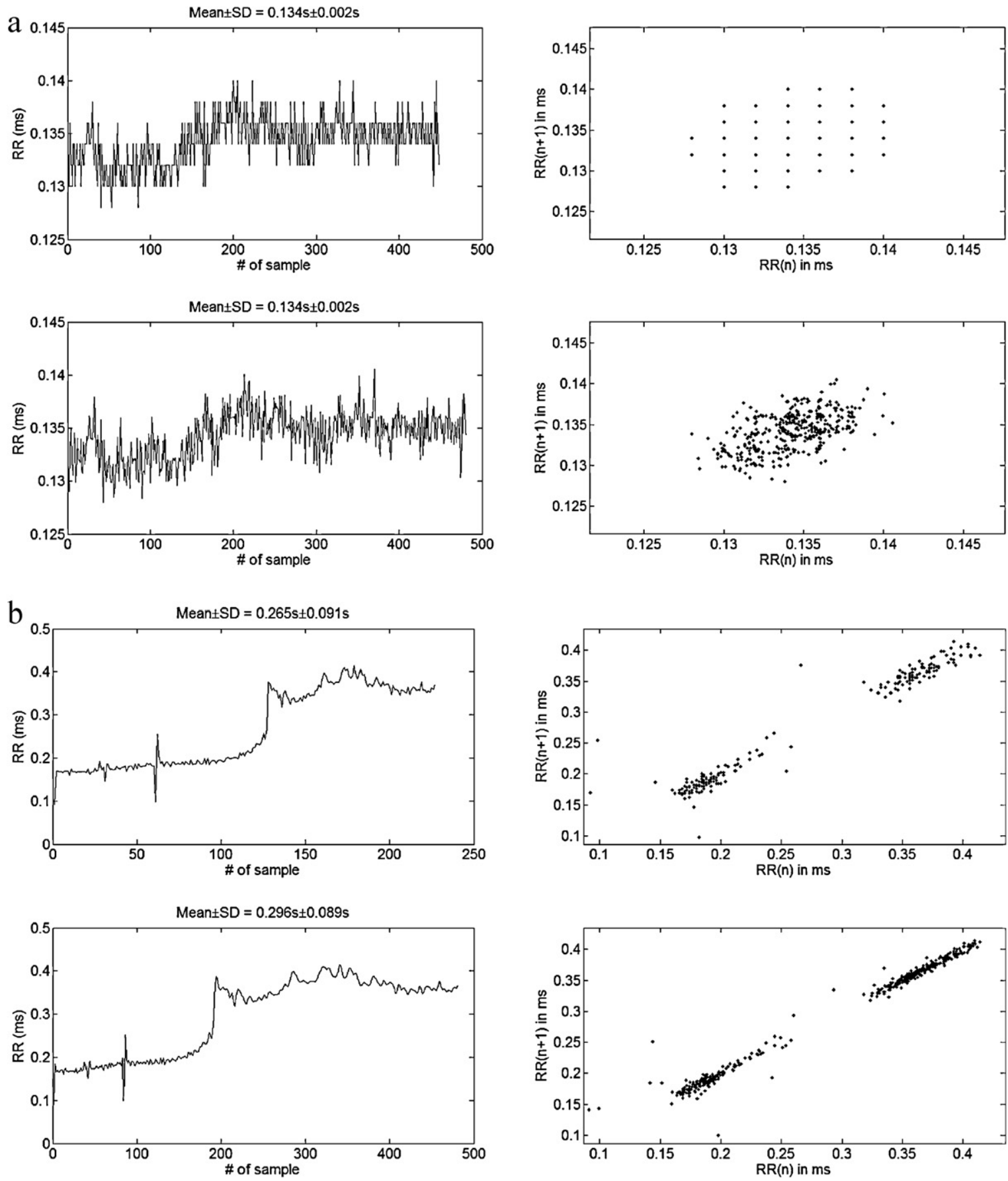

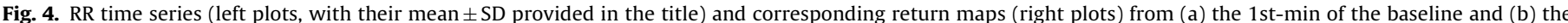

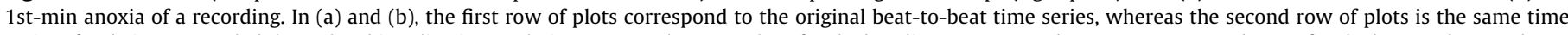

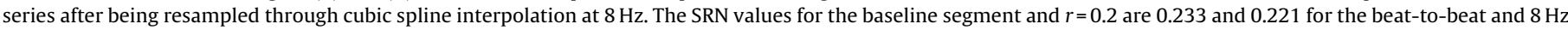
sampling, respectively. The SRN values for the anoxia segment and $r=0.2$ are 9.118 and 8.917 for the beat-to-beat and $8 \mathrm{~Hz}$ sampling, respectively.

ventilation $(\alpha=0.05)$. This supports the idea that the formulas for the selection of parameter $r$ proposed by Lu et al. [30] and Chon et al. [7] may not be adequate for the analysis of animal HR recordings. In addition, similar results were found using both Eqs. (7) and (8), and so only results considering Eq. (7) are presented in Table 2.

Significant differences between the different segments were found within linear time-domain indices, with each index representing different evolutions throughout the baseline, mechanical ventilation and anoxia periods (Table 1). On the other hand, frequency-domain indices showed limited ability to discriminate between different HR segments (Table 1 ), which may be related to the non-stationary nature of the recordings, as well as to outliers. These difficulties may be partly solved by a pre-processing algorithm, but such an algorithm might influence other HR parameters and thus its definition would require a study strictly devoted to the matter.

The HR index that better discriminated baseline (B1) and mechanical ventilation from anoxia was SampEn(2.0.6) (Table 2 and Fig. 5), despite its considerable inter-individual variation in the second (B2), third (B3) and fourth (B4) baseline segments (Fig. 5). A closer inspection of the relation between the $\operatorname{SampEn}(2,0.6)$ and the corresponding SRN values did not suggest any direct relation that 

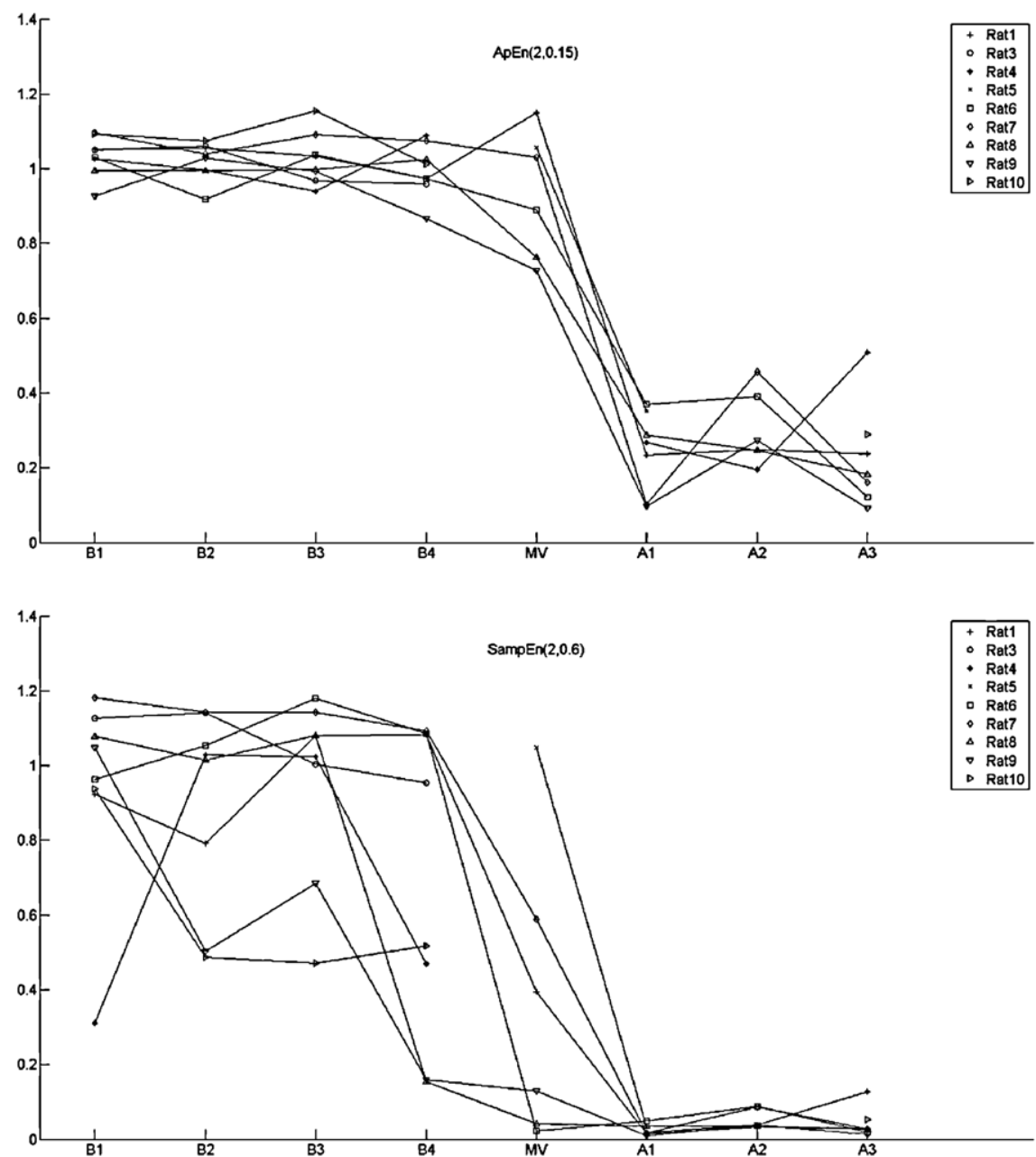

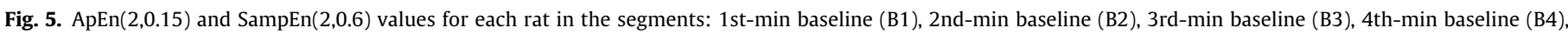

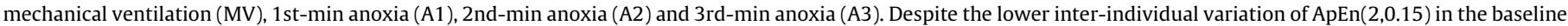
segments with respect to SampEn(2,0.6), the later index provides a better separation between B1 and MV.

could justify the increased inter-individual variation. ApEn $(2,0.15)$ presented lower inter-individual variation than $\operatorname{SampEn}(2,0.6)$, but it also achieved lower statistical discrimination of the HR segments (Fig. 5 and Table 2). Nevertheless, despite the inter-individual variation in baseline and mechanical ventilation segments, the bootstrap percentile 95\% confidence intervals presented in Tables 1 and 2 allowed for discriminating the different periods of the experimental protocol.

The loss of discriminating ability between baseline and anoxia periods as the scale increased in the MSE analysis, suggests that MSE may be less appropriate for the analysis of HR recordings that comprise rapid transitions between different acute pathological conditions (Fig. 6), and thus more suitable for the analysis of longer and stable segments. It can also be observed that as the scale increases, and consequently smaller segments are considered, there is an increase of the error bars motivated by the reduced length of the sequences associated with the higher scales.

\section{Discussion}

Linear and nonlinear HR analysis has been previously performed in a rat model of acute anoxia imposed on normal animals [19], as well as in a rat model of chronic pulmonary hypertension [20]. In this study, a far more critical situation, of acute anoxia superimposed on a model of chronic pulmonary hypertension was explored, with a particular attention devoted to novel entropy-based measures. Hopefully, this may help to further understand and develop HR monitoring systems for critical chronic patients during surgery or in intensive care units, as well as for compromised foetuses during labour.

In a previous work of our research group [20], we have compared the computed HRV indices obtained from the chronic pulmonary hypertension rats with those obtained from a normal group, in the range between weeks 0 and 4 of the experimental protocol. The acute anoxia event was imposed on week 5 of the chronic pulmonary hypertension rats, whereas it was performed on week 4 of the normal group [19]. Therefore, for this reason, and other factors previously addressed in [20], a direct comparison between the two acute anoxia events may not be adequate. Nevertheless, it was observed that the transition from baseline (and mechanical ventilation) to the first minute of anoxia is associated with a decrease in entropy indices in both groups. However, the normal group exhibited a slight increase of entropy indices in the third minute of anoxia [19], whereas the chronic pulmonary hypertension rats did not exhibit this type of "recovery" behaviour.

Female young adult rats were preferred at our laboratory although they are prone to estrous cycle variations - because they seem to be more sensitive to MCT than males [20]. Another important factor to mention relies on the type of instrumentation used to record HR, which implied anesthesia with Ketamine and rat manipulation, already addressed in our previous work [20]. Although we were aware of the disadvantages of this approach compared with 

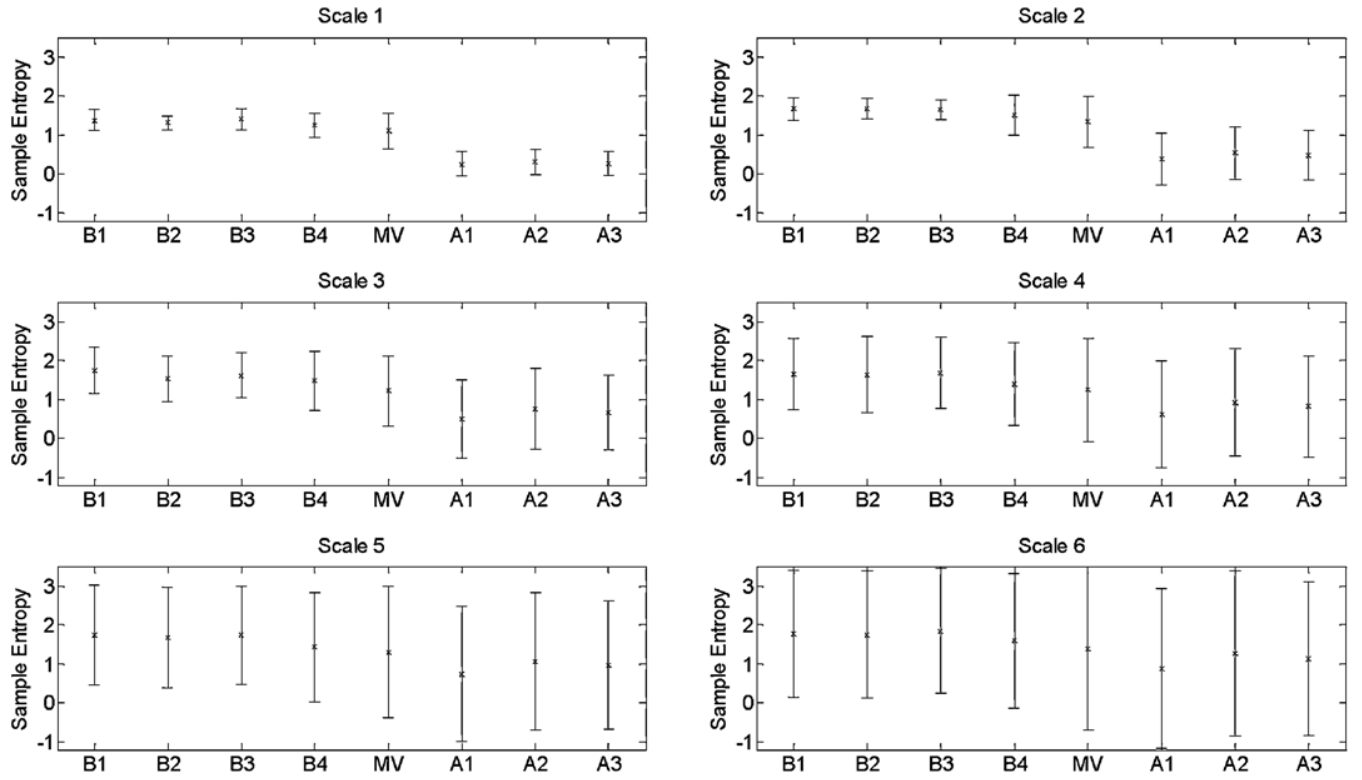

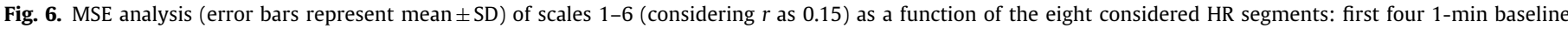
segments, mechanical ventilation and first three 1 -min segments of the anoxia period.

telemetry monitoring, for the particular objectives of our study, we did not have access to telemetry. Nevertheless, the laboratory conditions used in this study remain a standard procedure in many other animal experiments on heart research. Hopefully, in future studies this should be substituted by telemetric HR recording avoiding anesthesia and minimizing artificial rat manipulation and instrumentation.

As the quantification errors in the discrete RR time series may produce considerable errors in the ApEn estimation (bias and variance) when the signal variability or the sampling frequency is low [15], the influence of the quantization error on the computation of ApEn was analysed through the figure of merit SRN proposed by García-González et al. [15], which is a function of SDNN (SD of the RR sequence), the sampling frequency and the entropy threshold $r$. The resampling procedure may contribute to errors in ApEn estimation, namely by introducing bias. However, it is also associated with a monotonic decrease of error as SRN increases [15]. A proper comparison of ApEn indices, by taking into account similar experimental settings, may allow for controlling the error associated with resampling. Given the marked differences in mean and SD of HR signals between human and animal recordings, a reformulation of SRN according to the experimental settings may comprise the use of a different scattering measure such as the coefficient of variation.

In general, the results obtained in this study, regarding the detection of the effects of acute anoxia on the HR of rats with induced pulmonary hypertension, compared with the results obtained in a similar study with normal rats, suggest a similar performance of the entropy methods of HR analysis, but a worse performance of the linear methods [19]. The limited performance of frequency-domain indices in the discrimination of different HR episodes may be related to the non-stationary nature of HR. This problem may be elucidated by an adequate pre-processing algorithm - such as the algorithm proposed by Wessel et al. [42] - an hypothesis that certainly deserves further testing. Such an algorithm should also consider the removal of trends present in the signal, but this may also lead to an underestimation of entropy indices [27].

Obtaining accurate complexity measurement of the signal is especially desirable under circumstances in which a comparison is made between a baseline biological system state and a pathological condition or pharmacological manipulation [30]. Moreover, it was pointed out that further studies were needed to examine how the calculation of the maximum entropy value can help in improving classification and quantitative data analysis between normal and pathological states. In this work, some achievements regarding these considerations were obtained, with application to the HRV analysis in an animal model.

Lake et al. [27] have used a search strategy in order to select the most suitable values of parameters $m$ and $r$ in SampEn. They developed a systematic general approach to picking $m$ and $r$ based on a new metric of the efficiency of the entropy estimate. However, as assumed by the authors, the obtained values for a particular dataset may not be universally applicable to all datasets. Moreover, the proposed approach is computationally expensive. Nevertheless, it is known that ApEn is less sensitive to the choice of $m$ than $r$ [30], and thus the use of $m=2$ in our work was not a critical issue.

In previous studies, it was also found that it might be necessary to adequate the threshold $r$ in animal HRV studies, and additional values were proposed based on the coefficient of variation of animal HRV [19,20]. Recently, a set of formulas were developed for an automatic selection of the threshold value $r$ for ApEn [7,30], and were evaluated in the characterization of heart failure and healthy control groups [29]. To our knowledge, the present study is the first where those formulas were tested regarding its application to animal HRV data. One of the main advantages of this procedure is the fact that it avoids the highly time consuming task of searching for the $r$ parameter which maximizes the entropy measure. The similar conclusions obtained with formulas both from [7] and [30] support that their differences are due to random number generator.

Another limitation of ApEn and SampEn is the possibility of indicating higher entropy measures for white noise than for signals presenting some long-range dependence $[7,8,30]$. In the work of Lu et al. [30] and Chon et al. [7], they argued that it is due to the wrong selection of ApEn parameters, in particular due to the choice of the threshold $r$, and they propose equations for obtaining the most adequate parameter $r$, with respect to the embedding dimension $m$. On the other hand, Costa et al. [8] state that the paradox related to entropy measures is due to the fact of the traditional approach focus on a single scale. Accordingly, they proposed 
the multiscale entropy (MSE), where entropy is computed for several coarse-grained time series. Recently, a different viewpoint led to the development of a new entropy measure called Fuzzy Entropy (FuzzyEn), which replaces the 0-1 judgment of Heaviside function associated with ApEn and SampEn by a fuzzy function with defined properties, and also comprises the removal of the local baseline [4]. The removal of the local baseline may allow for minimizing the effect of non-stationarity in the time series. An alternative definition called Fuzzy Measure Entropy (FuzzyMEn) which accounts for both local and global baselines has also been proposed [28]. All these ideas deserve careful attention in HRV studies.

To our knowledge, MSE analysis has only been performed once in an acute animal model, with good results in the characterization of the respiratory response to the acute stimulation of the primary locus for respiratory rhythm generation [5]. However, in our study, there was a loss of discriminating ability between baseline and anoxia periods as the scale increased in the MSE analysis, suggesting that MSE may be less appropriate for the analysis of HR recordings that involve rapid transitions between different acute pathological conditions, related to anoxia (Fig. 6), as might occur, in clinical practice, during labour or surgery. Indeed, as the scale increased, an increase of the variance of the $\operatorname{SampEn}(2,0.15)$ measures was observed for all the HR segments (Fig. 6), explained by the non-stationary nature of the HR recordings but mostly by the relatively small length of the time series associated with the higher scales.

It is also important to put in evidence the difference between the concepts of complexity and regularity, since there is no straightforward correspondence between regularity and complexity [11]. Regularity is associated with the occurrence of repeated patterns, and can be measured by entropy-based algorithms, such as approximate and sample entropy measures [34,37]. On the other hand, complexity may be defined as a meaningful intricate arrangement of several parts of a system. Therefore, the concepts of regularity and complexity should not be mixed. For instance, considering the example of white noise measured with ApEn, it is associated with high irregularity and low complexity, whereas $1 / f$ noise (which contains complex structures) is associated with a lower irregularity measure. This reinforces the importance of a proper use of entropy measures when measuring the complexity of a system. In particular, the MSE approach allows for accounting for these issues in a relatively small number of time scales.

The MSE analysis is based on the computation of SampEn, considering the threshold value $r$ of scale 1 for all the other scales. This aspect was already object of discussion [10,33] and will deserve further attention in the future, namely under the scope of a wider evaluation of nonlinear methods for HR analysis. Other issue which will also deserve attention in the future is related to a sensitivity analysis of MSE analysis on the selection of threshold parameter $r$.

It is also known that the time delay may significantly influence the results obtained with ApEn and SampEn indices, due to the presence of long range correlation in HR signals [24]. A time delay equal to the first zero crossing or minimum of the autocorrelation function may provide consistent results, accounting for the presence of short or long range correlations present in the HR signal. This is of particular interest in situations associated with a slowly decaying autocorrelation function, such as those observed for the Lorenz and Rössler systems [24]. Therefore, this issue will deserve further research in the future.

Another factor that influences the computation of the entropy indices ApEn and SampEn is the presence of ectopic beats. Both entropy measures evaluate the irregularity of a sequence based on the distance between vectors, taking into account a threshold which relies on fixed parameter $(r)$ multiplied by the SD of the signal. Therefore, the presence of ectopic beats such as an extrasystole increases the SD of the signal, and consequently will lead to lower entropy values. However, their occurrence was estimated to be quite low, accomplished by relatively low amplitude, and thus its influence in the computation of entropy indices was minimal. Moreover, since ectopic beats may represent a 'typical' pattern in anoxia periods, their elimination may be controversial. These considerations support the interest on studying the effect of a preprocessing algorithm in this context.

The present work emphasizes the importance of an adequate selection of the entropy methods, as this may significantly influence the results. However, it is not possible at this point to indicate which is the best method - if there is one at all - as different combinations of measures/parameters may be associated with different physiological conditions. As shown in [11], the discrimination between healthy, congestive heart failure and atrial fibrillation groups is strongly influenced by the scale factor of the MSE. In particular, it is shown that scale 20 allows the distinction between the healthy and pathologic groups, and higher complexity values associated with the healthy group. However, at this scale the two pathologic groups overlap. On the other hand, other scales allow other discriminations. This reinforces the need of combining not only specific values of the entropy measure with the time scale, as well as the combination of different (linear and nonlinear) HR parameters. Classification procedures such as neural networks or decision trees may be adequate for this purpose, aiming to support the interest in further translational research.

This study has several limitations. The number of included cases was relatively small, limited by the availability of resources and ethical issues. Moreover, HR analysis was performed in anesthetized animals. This was an appropriate choice to study acute conditions arising in surgical or intensive care situations and allows sounder comparisons with other studies using similar methods $[19,20]$, but needs to be critically considered when extrapolating results to situations where anaesthesia was not used. Another possible limitation of the present work, already expressed in a previous paragraph, is related with the fact that ectopic beats have not been removed from the HR signal. Last but not least, although the results of this study may help to understand and manage similar clinical situations, any attempt of translation to human scenarios should be performed very carefully and confirmed in clinical studies.

Finally, the research works regarding the study of the optimum threshold $r$ for ApEn raise the question: what is actually more appropriate to discriminate signals with different characteristics, the $r$ value, the ApEn value or both? A systematic evaluation of all linear and nonlinear computed measures is certainly warranted, in order to identify which indices or combination of indices is more appropriate for HR analysis of different physiopathological conditions.

In conclusion, it was shown that methods of heart rate entropy analysis were able to identify the transition between different states of acute anoxia superimposed on a chronic rat model of pulmonary hypertension. The transition from baseline to acute anoxia was accompanied by a significant decrease of entropy indices, which remained extremely low during the anoxia period. Moreover, there were no signs of recovery to the previous baseline values in chronically diseased pulmonary hypertensive rats. Our results may provide some clues on how to evaluate heart rate variability under hypoxic conditions in chronic human disease and may assist the development of mathematical tools for early identification of hypoxia, one of the main mechanisms of acute deterioration in chronic disease that commonly leads to devastating and even fatal outcomes. Still, the application to clinical scenarios such as critical care, anaesthesia, surgical procedures and foetal peripartum monitoring will require further critical analysis of human data. An important finding is that issues such as sampling rate and entropy 
parameters should be well accounted for during the application of entropy methods.

\section{Competing interests}

None declared.

\section{Funding}

This study was supported by project POSI/CPS/40153/2001 from Fundação para a Ciência e Tecnologia, Portugal.

\section{Ethical approval}

Not required.

\section{Acknowledgement}

Hernâni Gonçalves thanks Fundação para a Ciência e a Tecnologia (FCT), Portugal, for the financial support (post-doctoral grant SFRH/BPD/69671/2010).

\section{References}

[1] Acharya UR, Joseph KP, Kannathal N, Lim CM, Suri JS. Heart rate variability: a review. Med Bio Eng Comput 2006;44:1031-51.

[2] Aubert AE, Vandeput S, Beckers F, Liu J, Verheyden B, Van Huffel S. Complexity of cardiovascular regulation in small animals. Philos T R Soc A 2009;367:1239-50.

[3] Cerutti C, Gustin MP, Paultre CZ, Lo M, Julien C, Vincent M, et al. Autonomic nervous-system and cardiovascular variability in rats - a spectral-analysis approach. Am J Physiol 1991;261:H1292-9.

[4] Chen W, Zhuang J, Yu W, Wang Z. Measuring complexity using FuzzyEn, ApEn and SampEn. Med Eng Phys 2009;31:61-8.

[5] Chen X, Chon KH, Solomon I. Chemical activation of pre-Bötzinger complex in vivo reduces respiratory network complexity. Am J Physiol Regul Integr Comp Physiol 2005;288:R1237-47.

[6] Chialvo DR. Unhealthy surprises. Nature 2002;419:263.

[7] Chon KH, G: Scully C, Lu S. Approximate entropy for all signals. Is the recommended threshold value $r$ appropriate? IEEE Eng Med Biol 2009;28:18-23.

[8] Costa M, Goldberger AL, Peng CK. Multiscale entropy analysis of complex physiologic time series. Phys Rev Lett 2002;89:068102.

[9] Costa M, Goldberger AL, Peng CK. Multiscale entropy to distinguish physiologic and synthetic RR time series. Comput Cardiol 2002;29:137-40.

[10] Costa M, Goldberger AL, Peng CK. Reply to Comment on "Multiscale entropy analysis of complex physiologic time series". Phys Rev Lett 2004;92:089804.

[11] Costa M, Goldberger AL, Peng CK. Multiscale entropy analysis of biological signals. Phys Rev E 2005;71:021906.

[12] Dudewicz E, Mishra S. Modern mathematical statistics. New York: Wiley; 1988.

[13] Eckmann JP, Ruelle D. Ergodic theory of chaos and strange attractors. Rev Mod Phys 1985;57:617-56.

[14] Fusheng Y, Bo H, Qingyu T. Approximate entropy and its application in biosignal analysis. In: Akay MM, editor. Nonlinear biomedical signal processing - volume II: dynamical analysis and modeling. IEEE Press; 1998. p. 72-91.

[15] García-González MA. Fernández-Chimeno M, Ramos-Castro J. Errors in the estimation of approximate entropy and other recurrence-plot-derived indices due to the finite resolution of RR time series. IEEE T Bio-Med Eng 2009;56:345-51.

[16] Gonçalves H, Rocha AP, Ayres-de-Campos D, Bernardes J. Internal versus external intrapartum foetal heart rate monitoring: effect on linear and nonlinear parameters. Physiol Meas 2006;27:307-19.

[17] Gonçalves H, Rocha AP, Ayres-de-Campos D, Bernardes J. Linear and nonlinear fetal heart rate analysis of normal and acidemic fetuses in the minutes preceding delivery. Med Bio Eng Comput 2006;44:847-55.
[18] Gonçalves H, Bernardes J, Rocha AP, Ayres-de-Campos D. Linear and nonlinear analysis of heart rate patterns associated with fetal behavioral states in the antepartum period. Early Hum Dev 2007;83:585-91.

[19] Gonçalves H, Henriques-Coelho T, Bernardes J, Rocha AP, Nogueira A, LeiteMoreira A. Linear and nonlinear heart rate analysis in a rat model of acute anoxia. Physiol Meas 2008;29:1133-43.

[20] Gonçalves H, Henriques-Coelho T, Bernardes J, Rocha AP, Brandão-Nogueira A, Leite-Moreira A. Analysis of heart rate variability in a rat model of induced pulmonary hypertension. Med Eng Phys 2010;32:746-52.

[21] Grassberger P, Procaccia I. Estimation of the Kolmogorov entropy from a chaotic signal. Phys Rev A 1983;28:2591-3.

[22] Hayes M. Statistical digital signal processing and modeling. John Wiley and Sons Inc; 1996.

[23] Ho KK, Moody GB, Peng CK, Mietus JE, Larson MG, Levy D, et al. Predicting survival in heart failure case and control subjects by use of fully automated methods for deriving nonlinear and conventional indices of heart rate dynamics. Circulation 1997;96:842-8.

[24] Kaffashi F, Foglyano R, Wilson CG, Loparo KA. The effect of time delay on Approximate \& Sample Entropy calculations. Physica D 2008;237:3069-74.

[25] Kolmogorov AN. A new metric invariant of transient dynamical systems and automorphisms in Lebesgue spaces. Dokl Akad Nauk SSSR+1958;119:861-4.

[26] Kuwahara M, Yayou K, Ishii K, Hashimoto S, Tsubone H, Sugano S. Power spectral analysis of heart rate variability as a new method for assessing autonomic activity in the rat. J Electrocardiol 1994;27:333-7.

[27] Lake DE, Richman JS, Griffin MP, Moorman JR. Sample entropy analysis of neonatal heart rate variability. Am J Physiol Regul Integr Comp Physiol 2002;283:R789-97.

[28] Liu C, Zhao L. Using fuzzy measure entropy to improve the stability of traditional entropy measures. Comput Cardiol 2011;38:681-4.

[29] Liu CY, Liu CC, Shao P, Li L, Sun X, Wang X, et al. Comparison of differen threshold values $r$ for approximate entropy: application to investigate the heart rate variability between heart failure and healthy control groups. Physiol Meas 2011;32:167-80.

[30] Lu S, Chen X, Kanters JK, Solomon IC, Chon KH. Automatic selection of the threshold value $r$ for approximate entropy. IEEE T Bio-Med Eng 2008:55:1966-72.

[31] Martinez WL, Martinez AR. Computational statistics handbook with MATLAB Boca Raton, FL: CRC Press; 2002.

[32] Moraru L, Cimponeriu L, Tong S, Thakor N, Bezerianos A. Characterization of heart rate variability changes following asphyxia in rats. Method Inform Med 2004;43:118-21

[33] Nikulin VV, Brismar T. Comment on "Multiscale Entropy Analysis of Complex Physiologic Time Series". Phys Rev Lett 2004;92:089803.

[34] Pincus S. Approximate entropy as a measure of system complexity. Proc Nat Acad Sci USA 1991;88:2297-301.

[35] Pincus SM, Goldberger AL. Physiological time-series analysis: what does regularity quantify? Am J Physiol 1994;266:H1643-56.

[36] Pincus S. Approximate entropy (ApEn) as a complexity measure. Chaos 1995:5:110-7.

[37] Richman JS, Moorman JR. Physiological time-series analysis using approximate entropy and sample entropy. Am J Physiol Heart Circ Physiol 2000;278:H2039-49.

[38] Shannon CE. A mathematical theory of communication. Bell Syst Tech J 1948;27:379-423.

[39] Shaw R. Strange attractors, chaotic behavior, and information flow. Z Naturforsch A 1981;36:80-112.

[40] Signorini MG, Magenes G, Cerutti S, Arduini D. Linear and nonlinear parameters for the analysis of fetal heart rate signal from cardiotocographic recordings. IEEE T Bio-Med Eng 2003;50:365-74.

[41] Task force of the European Society of Cardiology and the North American Society of Pacing and Electrophysiology. Task Force Heart rate variability: standards of measurement, physiological interpretation and clinical use. Circulation 1996;93:1043-65.

[42] Wessel N, Voss A, Malberg H, Ziehmann C, Voss HU, Schirdewan A, et al. Nonlinear analysis of complex phenomena in cardiological data. Herzschr Elektrophys 2000;11:159-73.

[43] Yatang L, Jiaheng Q, Zhuo Y, Johns EJ, Tao Z. Long-range correlation of rena sympathetic nerve activity in both conscious and anesthetized rats. J Neurosci Meth 2008;172:131-6 